\title{
Análisis de la participación en encuestas de cinco países: relevancia para la investigación en salud pública
}

\author{
Armando Peruga, ${ }^{1}$ Esther María León, ${ }^{2}$ Raquel Child, ${ }^{3}$ Aurelio \\ Cruz, ${ }^{4}$ Manuel Hernández, ${ }^{5}$ Anabella Arredondo, ${ }^{3}$ \\ Carlos Hernández, ${ }^{4}$ Paloma Cuchí ${ }^{6}$ y Fernando Zacarías ${ }^{1}$
}

RESUMEN Se presenta una comparación de las tasas de participación y de las razones de la falta de respuesta en encuestas realizadas en cinco países de América Latina y el Caribe con el objetivo de medir la prevalencia de comportamientos de riesgo que determinan la transmisión del virus de la inmunodeficiencia humana. Dichas encuestas se basaron en muestras probabilísticas de la población de ambos sexos comprendida entre 15 y 49 años de edad, excepto en México, donde se estudió solo a los hombres. Se estimaron tres componentes de la participación: las proporciones de viviendas entrevistadas, de viviendas entrevistadas con personas elegibles y de personas elegibles que cumplimentaron la entrevista. Además, se calculó un índice global que combinaba los tres componentes. La tasa global de respuesta osciló entre 35,6\% en México y 81,4\% en Chile, y el componente de esta tasa con mayor variabilidad fue la participación de personas elegibles, que varió entre $50 \%$ en México y 95\% en Cuba. Estos valores fueron más bajos de lo esperado, sobre todo en los hombres, y servirán de orientación para futuras encuestas, ya que se deberán considerar tasas de rechazo mayores de las previstas en el protocolo. Los resultados permiten inferir la validez de las estimaciones de la prevalencia de los diversos comportamientos de riesgo observados y establecen una referencia para calcular el tamaño muestral de futuras encuestas y mejorar la metodología de la investigación.

Dos revisiones $(1,2)$ de la literatura científica publicada en América Latina

\footnotetext{
Organización Panamericana de la Salud, Washington, DC, Estados Unidos de América. Toda la correspondencia debe ser enviada a Armando Peruga, a la siguiente dirección postal: $\mathrm{PAHO}, \mathrm{HCN}$ room 416, 525 23rd St. NW, Washington, D.C. 20037, USA.

2 Oficina Nacional de Estadísticas de Cuba, La Habana, Cuba.

3 Comisión Nacional del SIDA de Chile, Santiago, Chile.

4 Instituto Nacional de Salúd Pública de México, Cuernavaca, México.

5 Ministerio de Salud Pública de Cuba, La Habana, Cuba.

6 ONUSIDA, Washington, D.C., Estados Unidos de América.
}

en la última década, la primera sobre el conjunto de artículos de salud pública y la segunda acerca de la investigación sobre tabaco, indicaron que más de 95\% de los estudios cuantitativos originales eran encuestas con un diseño transversal. La validez de los resultados de estas encuestas viene determinada, en gran parte, por la proporción de personas seleccionadas que participan en el estudio. Muchos de estos estudios, sin embargo, no informaban de las tasas de participación o, si lo hacían, no daban detalles de los componentes de la falta de participación que permitieran explicarla y me- jorar la metodología de futuras encuestas y, por ende, la calidad de la investigación en salud pública.

En este artículo se presenta una comparación de las tasas de participación y de las razones de la falta de respuesta en cinco países de América Latina que realizaron la misma encuesta sobre comportamientos sexuales de riesgo. La encuesta se basó en una metodología estandarizada diseñada en 1994 por el entonces Programa Mundial del SIDA de la Organización Mundial de la Salud para ayudar a los programas nacionales a evaluar el impacto de sus actividades preventivas (3). Esta meto- 
dología constaba de tres protocolos. El primero tenía como objetivo evaluar la calidad de la atención clínica a pacientes con enfermedades de transmisión sexual y ha sido aplicado en Jamaica (4). El segundo permitía producir datos para estimar la disponibilidad de preservativos para distribución en el ámbito central y periférico. El último era un protocolo para realizar encuestas repetidas de la población general adulta con el objetivo de medir la prevalencia de dos comportamientos de riesgo determinantes de la transmisión del virus de la inmunodeficiencia humana: el número de parejas sexuales y la ausencia de uso de preservativos durante la relación sexual.

Este tercer protocolo fue aplicado en cinco países de América Latina y el Caribe entre 1995 y 1996: Chile, Costa Rica, Cuba, México y República Dominicana. Los resultados que se presentan en este artículo son importantes para interpretar las estimaciones de la prevalencia de los diversos comportamientos de riesgo observados y establecen una referencia para calcular el tamaño muestral de futuras encuestas en estos $u$ otros países que estén contemplando repetir o comenzar evaluaciones similares y para mejorar la metodología de la investigación.

\section{MATERIALES Y MÉTODOS}

En los cinco países se investigaron los comportamientos de riesgo de la población de ambos sexos comprendida entre 15 y 49 años de edad que residían en viviendas particulares, excepto en México, donde se estudió solo a los hombres. Las encuestas de los cinco países se basaron en dos muestras probabilísticas, una para cada sexo, excepto en Chile, donde se seleccionó una sola muestra para los dos sexos, y en México, donde se seleccionaron solamente hombres.

Las muestras fueron diseñadas en cada país de acuerdo con sus características y con los recursos cartográficos y de información actualizada disponible. Las estrategias muestrales adoptadas se resumen en el cuadro 1 . Se utilizó un muestreo por conglomerados multietá- pico estratificado. Dentro de cada conglomerado se seleccionaron las viviendas y se intentó entrevistar a alguno de los residentes para comprobar la existencia de personas elegibles para la encuesta. Una vez identificadas las personas elegibles en cada vivienda, se listaron y se eligió aleatoriamente a una de ellas para entrevistar. En Costa Rica, sin embargo, el listado de elegibles se elaboró para cada conglomerado en su conjunto, por lo que la vivienda no constituyó una unidad de muestreo. El cuadro 2 muestra las unidades y etapas muestrales de cada encuesta.

La entrevista se realizó cara a cara por visita directa a la vivienda, sin reemplazo de viviendas ni de personas. El entrevistador intentó cumplimentar el cuestionario al menos en tres ocasiones diferentes si, en sucesivas visitas al domicilio con cita previa, la persona seleccionada no estaba disponible o estaba ausente.

Los tres grandes grupos de causas por las que se podía producir una reducción de la muestra o disminución de la participación fueron los siguientes:

1. La imposibilidad de entrevistar a alguien en las viviendas seleccionadas a partir del marco muestral disponible para comprobar si existían personas elegibles, bien porque se tratase de direcciones incorrectas o inexistentes, o bien porque fuesen domicilios no residenciales, viviendas vacías o viviendas con residentes ausentes durante un largo período de tiempo o residentes que no colaboraron en esta etapa del muestreo. Este tipo de participación se estimó mediante lo que denominamos tasa de participación de viviendas y se calculó como el porcentaje de viviendas entrevistadas sobre el total de viviendas seleccionadas.

2. La inexistencia de personas del sexo y edad elegible en el domicilio seleccionado y entrevistado. La participación en esta fase se estimó mediante la tasa de elegibilidad, calculada como el porcentaje de viviendas con personas elegibles sobre el total de viviendas entrevistadas.

3. La negación expresa de las personas elegibles a contestar a la encuesta, la imposibilidad de entrevistarlas por ausencia reiterada del domicilio en el momento de la entrevista y otras causas de no participación de las personas elegibles. Estas tres causas se tabularon separadamente y se calculó la tasa de participación de personas elegibles: porcentaje de personas que cumplimentaron la entrevista sobre el total de personas elegibles seleccionadas para ser entrevistadas.

Los datos de cada estudio nacional fueron analizados con el programa estadístico SPSS (5).

\section{RESULTADOS}

El cuadro 3 muestra la participación de viviendas y personas por país. La tasa de participación de viviendas fue superior a 90\% en Costa Rica y Cuba, y cercana a $80 \%$ en México y la República Dominicana; en Chile, no se pudo calcular porque no se registraron los datos suficientemente desglosados por cada fase del levantamiento de la encuesta y, por tanto, se presenta la tasa de participación de viviendas y de elegibilidad conjuntamente.

La tasa de elegibilidad fue superior a $80 \%$ en México y en la República Dominicana, mientras que en Costa Rica sobrepasó $70 \%$ y en Cuba no superó dicha cifra. La tasa combinada de participación de viviendas y elegibilidad fue superior a $95 \%$ en Chile, donde hubo una sola muestra para ambos sexos.

La tasa de participación de personas elegibles osciló ampliamente entre 50\% en México y casi $95 \%$ en Cuba. En Costa Rica, Cuba y República Dominicana la principal causa de no participación fue la ausencia reiterada del domicilio de la persona elegible seleccionada, mientras que en el resto de los países fue la negativa a participar en la encuesta.

En el cuadro 4 se indica el número de entrevistados en comparación con el número de personas que se calculó que eran necesarias para alcanzar un tamaño muestral adecuado para obtener resultados válidos. Con la excep- 
CUADRO 1. Resumen de la estrategia muestral en cada país

\begin{tabular}{|c|c|c|c|c|c|}
\hline & Chile & Costa Rica & Cuba & México & República Dominicana \\
\hline Alcance territorial & $\begin{array}{l}\text { Áreas urbanas de } \\
\text { Santiago, Valparaíso y } \\
\text { Viña del Mar }\end{array}$ & $\begin{array}{l}\text { Área urbana de todo } \\
\text { el país }\end{array}$ & Ciudad de la Habana & Ciudad de México & Todo el país \\
\hline Trabajo de campo & Enero-abril, 1996 & Abril-mayo, 1996 & Enero-febrero, 1996 & Octubre-diciembre 1994 & Abril-junio, 1996 \\
\hline Marco muestral & $\begin{array}{l}\text { MMMa de la Dirección } \\
\text { General de Estadísti- } \\
\text { cas (información carto- } \\
\text { gráfica y numérica) }\end{array}$ & $\begin{array}{l}\text { MMM de la Dirección } \\
\text { General de } \\
\text { Estadísticas y Censos } \\
\text { (1984, actualizado) }\end{array}$ & $\begin{array}{l}\text { MMM de la Oficina } \\
\text { Nacional de } \\
\text { Estadística (1995) }\end{array}$ & $\begin{array}{l}\text { MMM de la Dirección } \\
\text { General de } \\
\text { Epidemiología }\end{array}$ & $\begin{array}{l}\text { MMM del CPVb de la } \\
\text { Dirección Nacional de } \\
\text { Estadística (1993) }\end{array}$ \\
\hline Método de muestreo & $\begin{array}{l}\text { Muestreo por } \\
\text { conglomerado } \\
\text { cuatrietápico } \\
\text { estratificado }\end{array}$ & $\begin{array}{l}\text { Muestreo por } \\
\text { conglomerado } \\
\text { trietápico estratificado }\end{array}$ & $\begin{array}{l}\text { Muestreo por } \\
\text { conglomerado } \\
\text { trietápico estratificado }\end{array}$ & $\begin{array}{l}\text { Muestreo por } \\
\text { conglomerado } \\
\text { cuatrietápico } \\
\text { estratificado }\end{array}$ & $\begin{array}{l}\text { Muestreo por } \\
\text { conglomerado } \\
\text { cuatrietápico } \\
\text { estratificado }\end{array}$ \\
\hline Estratos geográficos & $\begin{array}{l}\text { Santiago y Región de } \\
\text { Valparaíso-Viña }\end{array}$ & $\begin{array}{l}\text { Área metropolitana de } \\
\text { San José, resto ur- } \\
\text { bano del Valle Central } \\
\text { y resto urbano del país }\end{array}$ & $\begin{array}{l}\text { Los } 15 \text { municipios de la } \\
\text { Habana }\end{array}$ & $\begin{array}{l}\text { Las } 16 \text { delegaciones } \\
\text { distritales }\end{array}$ & $\begin{array}{l}\text { Distrito Nacional y } \\
\text { Provincias divididos en } \\
\text { zonas urbana y rural }\end{array}$ \\
\hline $\begin{array}{l}\text { Estratos } \\
\text { socioeconómicos }\end{array}$ & Alto, medio y bajo & No se consideraron & No se consideraron & No se consideraron & No se consideraron \\
\hline $\begin{array}{l}\text { Afijación de la } \\
\text { muestra por estrato }\end{array}$ & $\begin{array}{l}\text { Desproporcionada por } \\
\text { estrato }\end{array}$ & $\begin{array}{l}\text { Proporcional al tamaño } \\
\text { de cada estrato }\end{array}$ & $\begin{array}{l}\text { Proporcional al tamaño } \\
\text { de cada estrato }\end{array}$ & $\begin{array}{l}\text { Proporcional al tamaño } \\
\text { de cada estrato }\end{array}$ & $\begin{array}{l}\text { Proporcional al tamaño } \\
\text { de cada estrato }\end{array}$ \\
\hline $\begin{array}{l}\text { Probabilidades de } \\
\text { selección }\end{array}$ & $\begin{array}{l}\text { Una muestra } \mathrm{Pl}^{\mathrm{C}} \text { de } \\
\text { selección en cada } \\
\text { etapa de muestreo }\end{array}$ & $\begin{array}{l}\text { Dos submuestras } \\
\text { independientes } \\
\text { autoponderadas } \\
\text { (PSFI) }^{d}\end{array}$ & $\begin{array}{l}\text { Dos muestras } \\
\text { independientes } \\
\text { autoponderadas } \\
\text { (PSFI) }\end{array}$ & $\begin{array}{l}\text { Una muestra } \\
\text { autoponderada (PSFI) }\end{array}$ & $\begin{array}{l}\mathrm{PPT}^{\mathrm{e}} \text { en la primera } \\
\text { etapa y PI en las } \\
\text { restantes. Dos } \\
\text { submuestras }\end{array}$ \\
\hline Post-estratificación & $\begin{array}{r}\text { Sexo, edad y } \\
\text { escolaridad }\end{array}$ & Sexo & Sexo y edad & Edad & Sexo \\
\hline Método de estimación & Factores de corrección & $\begin{array}{l}\text { Estimadores de razón } \\
\text { separada }\end{array}$ & $\begin{array}{l}\text { Estimadores de razón } \\
\text { separada }\end{array}$ & $\begin{array}{l}\text { Estimadores de razón } \\
\text { separada }\end{array}$ & $\begin{array}{l}\text { Estimadores de razón } \\
\text { separada }\end{array}$ \\
\hline
\end{tabular}

ción de Cuba, los restantes cuatro países obtuvieron un tamaño muestral de varones inferior al esperado. De los cuatro países que seleccionaron una muestra de mujeres, dos (Costa Rica y República Dominicana) obtuvieron un tamaño muestral inferior al esperado.

\section{DISCUSIÓN}

Los diseños muestrales probabilísticos aplicados en todos los países, los tamaños muestrales finalmente alcanzados y los procedimientos de estima- ción utilizados permiten garantizar que las estimaciones fundamentales de la encuesta tienen la precisión estadística requerida. Sin embargo, la existencia de hogares y personas seleccionadas que no participaron en la encuesta abre la posibilidad de que exista un sesgo de participación debido a que los participantes sean diferentes de los no participantes en lo que se refiere a los comportamientos medidos. Los datos disponibles no permiten comprobar si dicha diferencia existe $(6,7)$. Tampoco conocemos datos que permitan comparar las tasas de participación de este es- tudio con las de trabajos similares. Sin embargo, la tasa global de respuesta en Chile es razonablemente alta. Por otra parte, la tasa global de respuesta en Costa Rica, Cuba y República Dominicana es menor que en Chile pero, en estos países, un componente importante de la reducción de la muestra fue la falta de personas elegibles en las viviendas seleccionadas, que es un factor incontrolable en el tipo de muestreo utilizado y no afecta a la representatividad de la muestra en tanto que no crea una selección diferenciada de participantes y no tiene potencial para 
CUADRO 2. Unidades muestrales en cada etapa de la selección por país

\begin{tabular}{|c|c|c|c|c|c|}
\hline Etapa & Chile & Costa Rica & Cuba & México & República Dominicana \\
\hline Segunda & Grupo de manzanas & Segmento compacto & Vivienda & Manzana & Segmento \\
\hline Cuarta & Individuo & - & - & Individuo & Individuo \\
\hline
\end{tabular}

crear un sesgo de participación. Además, la tasa de participación de viviendas en el presente estudio es similar en Cuba, y ligeramente inferior en Costa Rica y República Dominicana, a las obtenidas en otros estudios $(8,9)$. La tasa de participación de viviendas es indicativa de la calidad del marco muestral. En Costa Rica y Cuba esta tasa fue muy alta, posiblemente porque dispusieron de marcos muestrales maestros muy actualizados. En México y República Dominicana esta tasa fue menor, lo que pone de relieve la necesidad de una actualización cartográfica antes de emprender una encuesta de estas características.

La tasa de elegibilidad de estos dos últimos países parece ser algo inferior a la de otras encuestas, pero estos estudios no son totalmente comparables y no se pueden sacar conclusiones definitivas $(8,9)$. Por tanto, en Chile, Costa Rica, Cuba y República Dominicana, las tasas de participación obtenidas no permiten suponer que la prevalencia estimada de los comportamientos de riesgo deba interpretarse con mayor o menor cuidado que las de otras encuestas en lo que hace referencia a la representatividad de la muestra. Por el contrario, en México la tasa global de participación fue baja y, por tanto, no es necesariamente aplicable la misma suposición. Además, México ilustra la dificultad de la realización de encuestas en megalópolis, especialmente sobre temas potencialmente sensibles como son los comportamientos sexuales. Aunque no existen datos de encuestas comparables, las tasas de participación en algunos estudios realizados en el Distrito Federal de México fueron mayores que las obtenidas en nuestro estudio, sobre todo en lo que se refiere a la participación de personas elegibles $(10,11)$.

También cabe destacar que en los tres países (Chile, Costa Rica y República Dominicana) que obtuvieron submuestras de estratos geográficos diferenciados (urbanos de diferentes características o urbano y rural), la tasa global de respuesta no fue sustancialmente diferente entre ellos. Por tanto, no cabe suponer que haya efectos diferenciados de la participación sobre la representatividad de la muestra en cada uno de los estratos geográficos.

La tasa global de respuesta y las tres tasas que la componen son determinantes para calcular el tamaño muestral de futuras encuestas. La determi-

CUADRO 3. Participación de viviendas y personas por país

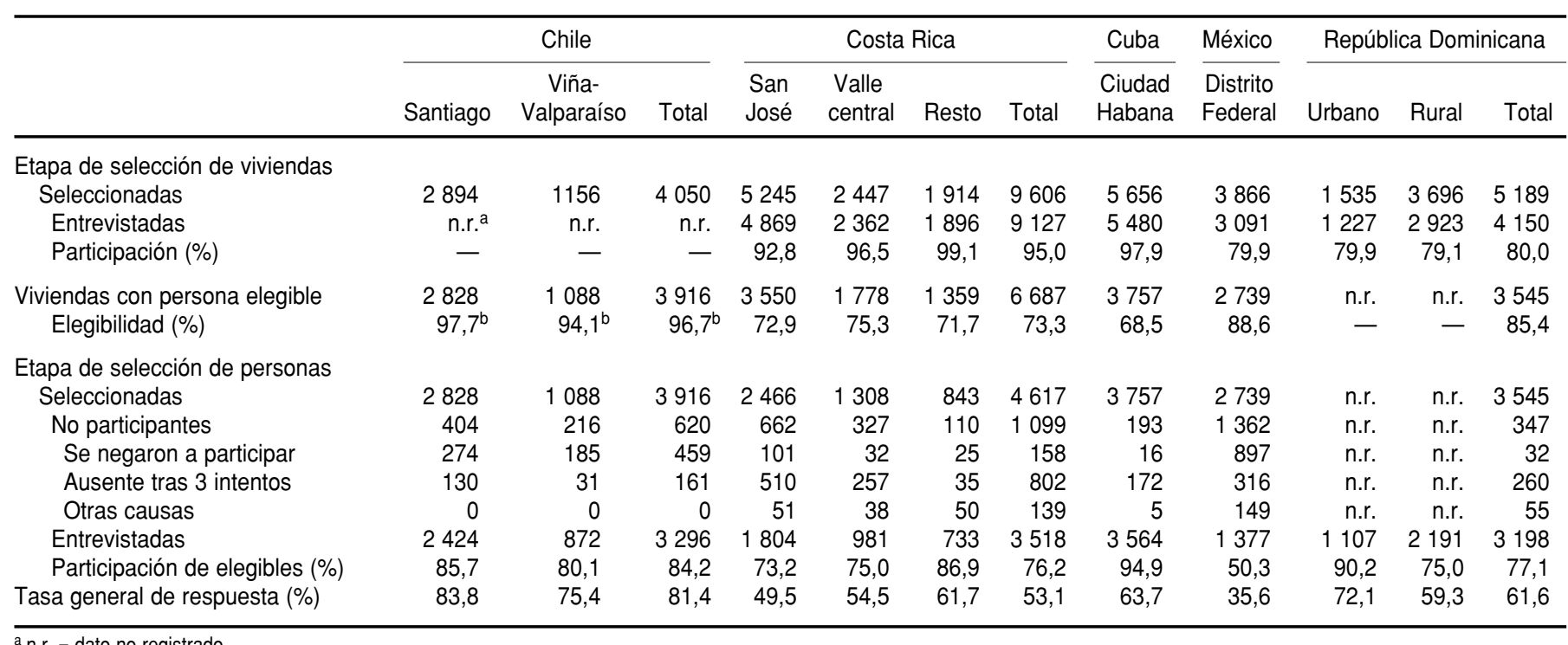

${ }^{a}$ n.r. = dato no registrado.

b Tasa combinada de participación de viviendas y elegibilidad. 
CUADRO 4. Proporción del tamaño muestral teórico alcanzado en cada país

\begin{tabular}{lccccc}
\hline & Chile & Costa Rica & Cuba & México & $\begin{array}{c}\text { República } \\
\text { Dominicana }\end{array}$ \\
\hline $\begin{array}{l}\text { Tamaño muestral teórico } \\
\quad \text { Mujeres }\end{array}$ & 1400 & 2000 & 1400 & 2000 & 2000 \\
$\quad \begin{array}{l}\text { Hombres } \\
\text { Entrevistados (No.) }\end{array}$ & 1400 & 2000 & 1400 & 2000 & 2000 \\
$\quad$ Mujeres & 1972 & 1839 & 1853 & - & 1784 \\
$\quad$ Hombres & 1324 & 1679 & 1711 & 1377 & 1414 \\
Entrevistados (\%) & 141 & 92 & 132 & - & 89 \\
$\quad$ Mujeres & 95 & 84 & 122 & 69 & 71 \\
$\quad$ Hombres & 118 & 88 & 127 & - & 80 \\
$\quad$ Ambos sexos & & & & & \\
\hline
\end{tabular}

nación del tamaño de la muestra se hace basándose en dos tipos de consideraciones: estadísticas, fundamentalmente la varianza esperada de las variables básicas y el efecto del diseño, y prácticas, fundamentalmente la participación esperada de viviendas y personas. Una de las dificultades que tuvieron las cinco encuestas a la hora de calcular el tamaño muestral fue precisamente la falta de información sobre la participación esperada en encuestas sobre comportamientos sexuales de riesgo, ya que era la primera vez que se realizaba un estudio de este tipo en la mayor parte de los países.

Los datos presentados indican que la tasa global de respuesta fue generalmente inferior a lo esperado cuando se hizo el cálculo muestral, sobre todo entre los hombres. Parte del problema radica en la selección de muestras independientes para hombres y mujeres, que reduce la probabilidad de encontrar una persona elegible en la vivienda seleccionada. En Chile, el único país donde se seleccionó una sola muestra para ambos sexos y el que presentó las tasas de elegibilidad más altas, se alcanzaron los tamaños muestrales originalmente previstos con solo un insignificante déficit entre los hombres. Por tanto, las próximas ediciones de esta encuesta deberán considerar la obtención de una sola muestra para ambos sexos.

El otro problema es que, excepto en Cuba, la participación de elegibles fue inferior a lo esperado, por debajo de $85 \%$. El rechazo explícito de los elegibles a participar fue más importante en Chile y México que en los otros tres países, donde la principal causa de falta de participación de los elegibles fue su ausencia repetida del domicilio, que impidió realizar la entrevista. En cualquier caso, este dato servirá de orientación para futuras encuestas en los cinco países de este estudio, ya que cuatro de ellos deberán considerar tasas de rechazo mayores de las previstas para este estudio. Para otros países, la única recomendación posible es que el cálculo del tamaño muestral de futuros estudios tome en consideración reducciones de la muestra similares a las más altas de las presentadas en este artículo.
Más allá de las conclusiones que se puedan extraer para repetir las encuestas analizadas, los resultados de este estudio ponen de manifiesto algunos aspectos que pueden mejorar metodológicamente la realización de investigaciones de salud pública en general, ya que la mayor parte de las que se hacen en América Latina y el Caribe se basan en diseños transversales como los usados aquí. En primer lugar, la experiencia del presente estudio muestra que la participación depende de la correcta y puntual actualización de los marcos muestrales maestros de cada país y de la calidad del trabajo de campo. En segundo lugar, es necesario fomentar la divulgación de los problemas encontrados para alcanzar la participación deseada tanto de viviendas como de sujetos seleccionados en las muestras de las encuestas (12). En este sentido, no basta con indicar la tasa de participación. Además, es necesario estudiar los componentes de la falta de participación para que otros científicos saquen lecciones para sus propias investigaciones. Por último, hay que entender mejor las razones de los encuestados para participar o no en los estudios, bien incorporando a las investigaciones en marcha variables y análisis que lo permitan o bien realizando estudios especiales.

La calidad metodológica de la investigación en salud pública es fundamental para asegurar que sus resultados sean válidos y útiles para mejorar la salud de las poblaciones. Dicha calidad no se puede fortalecer sin una profundización específica en los aspectos metodológicos, entre los que se encuentra el estudio de la participación, como el realizado aquí.

\section{REFERENCIAS}

1. Peruga A, Campillo C. Análisis de las publicaciones de salud pública en América Latina: 1989-1992. I Congreso Iberoamericano de Epidemiología. Granada 1995.

2. Peruga A, Robles $S$. La investigación sobre tabaco en las Américas. I Congreso Iberoamericano de Tabaco o Salud. Canarias, 1999.
3. Global Program on AIDS. Evaluation of a national AIDS programmme: a methods package for the prevention of HIV infection. Geneva: World Health Organization; 1994.

4. Bryce J, Vernon A, Brathwaite AR, Perry S, Figueroa JP, Emerson RB, and the Collabora- tive group on indicators of STD case-management. Quality of sexually transmitted disease services in Jamaica: evaluation of a clinicbased approach. Bull World Health Organ 1994;72:239-247.

5. SPSS for Windows 8.0. Chicago: SPSS Inc.; 1998 
6. Hill A, Roberts J, Ewings P, Gunnell D. Nonresponse bias in a lifestyle survey. J Public Health Med 1997;19:203-207.

7. Sudman S. Measuring behavior related to the risk of acquired immune deficiency syndrome. En Fowler FJ, ed. Health survey research methods. Washington: U.S. Department of Health and Human Services; 1989. p. 259.

8. CESDEM, Profamilia, ONAPLAN, Macro International Inc. República Dominicana: Encuesta demográfica y de salud, 1996. Calverton, MD: Macro International Inc.; 1997.
9. Madrigal J, Schifter J. Primera encuesta nacional sobre SIDA. San José de Costa Rica: Asociación Demográfica Costarricense; 1990.

10. México, Secretaría de Salud. Encuesta nacional de salud materno infantil. México, D.F.: Secretaría de Salud; 1994.

11. México, Secretaría de Salud e Institute for Resource Development/Macro Systems, Inc. Encuesta nacional sobre fecundidad y salud. 1987. Columbia, MD: Institute for Resource Development/Macro Systems, Inc.; 1989.

12. Ross S, Grant A, Counsell C, Gillespie W, Russell I, Prescott R. Barriers to participation in randomised controlled trials: a systematic review. J Clin Epidemiol 1999;52:1143-1156.

Manuscrito recibido el 28 de enero de 1999 y aceptado para publicación, tras revisión, el 8 de febrero de 2000

ABSTRACT This study compares participation rates and reasons for nonresponse in surveys conducted in five countries of Latin America and the Caribbean. The objective of the surveys was to measure the prevalence of risk behaviors affecting the transmission of human immunodeficiency virus. The surveys were based on probability samples of the population of both sexes between 15 and 49 years old, except in Mexico, where only men were included. Proportions of three components of participation were estimated: residences interviewed, interviewed residences with eligible persons, and eligible persons who completed the interview. In addition, an overall index that combined the three components was calculated. The overall response rate ranged from $35.6 \%$ in Mexico to $81.4 \%$ in Chile. The component with the greatest variability was the participation of eligible persons, which ranged from $50 \%$ in Mexico to $95 \%$ in Cuba. These values were lower than what had been expected, especially among men, and will serve to guide future surveys, since rejection rates higher than the ones expected in the protocol should be considered. The results make it possible to infer the validity of the prevalence estimates for the various observed risk behaviors. The results also establish a benchmark to calculate the sample size in future surveys and to improve research methodology. 